Pacific

Journal of

Mathematics

ON THE EXISTENCE OF INFINITELY MANY ESSENTIAL SURFACES OF BOUNDED GENUS

UlRich OeRTel 


\title{
ON THE EXISTENCE OF INFINITELY MANY ESSENTIAL SURFACES OF BOUNDED GENUS
}

\author{
ULRICH OERTEL
}

\begin{abstract}
A theorem of William Jaco and Eric Sedgwick states that if $M$ is an irreducible, $\partial$-irreducible 3 -manifold with boundary a single torus, and if $M$ contains no genus one essential (incompressible and $\partial$-incompressible) surfaces, then $M$ cannot contain infinitely many distinct isotopy classes of essential surfaces of uniformly bounded genus. The main result in this paper is a generalization: If $M$ is an irreducible $\partial$-irreducible 3-manifold with boundary, and $M$ contains no genus one or genus zero essential surfaces, then $M$ cannot contain infinitely many isotopy classes of essential surfaces of uniformly bounded genus.
\end{abstract}

\section{Introduction.}

In this paper, a Haken manifold is an orientable, irreducible, $\partial$-irreducible 3 manifold containing a (2-sided) incompressible surface. Even if a 3-manifold is not Haken, we shall always, for simplicity, assume that it is orientable. An irreducible, $\partial$-irreducible manifold $M$ is simple if it contains no incompressible, $\partial$-incompressible tori or annuli. We say a properly embedded 2 -sided surface $S \hookrightarrow M$ is essential if it is incompressible and $\partial$-incompressible and not a sphere or disc. In this paper all essential surfaces will be 2-sided and embedded, and it will be understood that "essential surface" means "isotopy class of an essential surface." The following is a well-known result attributed to Wolfgang Haken, see [7].

Theorem 1.1 (W. Haken). Let $M$ be a simple Haken manifold, then $M$ cannot contain infinitely many essential surfaces of uniformly bounded Euler characteristic $\chi$.

Here, in the introduction, we give sketchy explanation of the proof. For simplicity, we assume $M$ is closed. The proof uses normal surface theory. There are many sources: The original sources are [9] and [4]; an early source recommended for readability is [14]. For a more modern version in terms of triangulations, see for example [8] or [5]. To any given essential surface $S$ in a closed Haken 3-manifold $M$ equipped with a triangulation, one can associate an Euler ratio $|\chi(S)| / \operatorname{Area}(S)=\mathcal{R}(S)$. To measure area here, 
we first note that the surface $S$ can be isotoped to make it a normal surface relative to the triangulation; the area is then the area of the least area normal representative in the isotopy class of $S$, where the area is the usual the combinatorial measure of area defined in normal surface theory, i.e., the number of intersections of the surface with the 1-skeleton of the triangulation. The Euler ratio then measures the average "curvature per unit area" in the surface.

Now, given infinitely many distinct normal surfaces $S_{i}$, their areas must be unbounded, since there are just finitely many normal surfaces of a given area. If the Euler characteristics of these surfaces are uniformly bounded, $\left|\chi\left(S_{i}\right)\right| \leq U$ for all $i$, then the Euler ratios $\mathcal{R}\left(S_{i}\right)$ are not bounded away from 0 . After passing to a subsequence, $\mathcal{R}\left(S_{i}\right) \rightarrow 0$. There is a way of taking limits of (normalized) subsequences of the sequence $\left\{S_{i}\right\}$, which we shall describe in a later section. The limit is an embedded, measured, zeroEuler-characteristic lamination, which can be approximated by an embedded incompressible torus. The method of taking limits is well-known, see for example $[\mathbf{1 0}],[\mathbf{1 3}],[\mathbf{1 1}]$. This completes the sketch of the proof of the Haken result stated above.

We observe that the Euler ratio is closely related to the "isoperimetric ratio," see for example [11]. For a null homotopic curve $\gamma$ in $M$, the isoperimetric ratio is defined as $\operatorname{Area}(D) / \operatorname{Length}(\gamma)$ where $D$ is a least area null homotopy for $\gamma$ and length is also measured combinatorially. Spaces for which the isoperimetric ratios are uniformly bounded have negatively curved fundamental groups in the sense of Gromov, [3].

William Jaco and Eric Sedgwick have proved an interesting variation on the Haken finiteness theorem:

Theorem 1.2 (Jaco-Sedgwick). Let $M$ be an irreducible, D-irreducible 3manifold with boundary a single torus, and suppose $M$ contains no essential surfaces of genus 1 , then $M$ cannot contain infinitely many essential surfaces of uniformly bounded genus.

We shall give an easy proof of this result using branched surface techniques and the limiting argument mentioned above.

We give a further generalization to arbitrary Haken manifolds with boundary:

Theorem 1.3. Let $M$ be an irreducible, $\partial$-irreducible 3-manifold, and suppose $M$ contains no essential surfaces of genus 1 or genus 0 , then $M$ cannot contain infinitely many essential surfaces of uniformly bounded genus.

Returning to the original Haken finiteness theorem, we shall prove, as we have already mentioned, that in the case of a simple 3-manifold $M$ there is a constant $K$ such that any essential surface $S \hookrightarrow M$ satisfies $|\chi(S)| \geq K \operatorname{Area}(S)$. "Large area surfaces have large topology." This statement implies Theorem 1.1 and is only slightly stronger. When we measure 
the area of an essential surface, we assume, as usual, that we have chosen a triangulation for $M$ and that $\operatorname{Area}(S)$ means the area of a least area normal surface in the isotopy class of $S$.

In view of the Jaco-Sedgwick result, one might then ask whether a similar result is true in a manifold $M$ with boundary one torus and no genus one essential surfaces, whether "large area connected essential surfaces have large genus." This is true. We will prove the following stronger version of the JacoSedgwick theorem. Given an essential surface $S \hookrightarrow M$, a natural modified Euler ratio is $\overline{\mathcal{R}}=|\chi(\bar{S})| / \operatorname{Area}(S)$, where $\bar{S}$ is obtained from $S$ by capping all boundary components with discs.

Theorem 1.4. Let $M$ be a Haken 3-manifold with boundary a single torus and with a given triangulation. Suppose $M$ contains no genus one essential surfaces. Then there is a uniform lower bound $K>0$ for the Euler ratio $\overline{\mathcal{R}}(S)$ on all essential surfaces $S$.

We also have a slightly stronger version of Theorem 1.3:

Theorem 1.5. Let $M$ be a Haken 3-manifold and with a given triangulation. Suppose $M$ contains no genus one or genus zero essential surfaces. Then there is a uniform lower bound $K>0$ for the Euler ratio $\overline{\mathcal{R}}(S)$ on all essential surfaces $S \hookrightarrow M$.

\section{Preliminaries.}

We give here a brief informal overview of normal surfaces and incompressible or essential branched surfaces. There are numerous sources for these related subjects. We note that the earlier versions of normal surface theory were described in terms of handle decompositions, whereas the currently used normal surface theory is in terms of triangulations. In fact, the latter can be regarded as a special case of the former. For normal surface theory, see for example [4], [12], [5]; for branched surfaces as applied to incompressible surfaces and essential laminations see [1], [12], [2].

The basic result in normal surface theory says that any essential surface $S$ in a Haken 3-manifold $M$ can be isotoped to be normal relative to a triangulation. The same result applies to other essential surfaces, for example to essential spheres in reducible 3-manifolds, but we will not need these results. To say that an embedded surface is normal means that it intersects each 3-simplex of the triangulation in arbitrarily many discs, each combinatorially isomorphic to one shown in Figure 1. For each 3-simplex there are seven types of discs as in Figure 1 which are known as disc types, so that for a triangulation with $n 3$-simplices, there are $7 n$ disc types. Typically one goes further and chooses a normal representative of least area for the isotopy class of $S$, where $\operatorname{Area}(S)$ is the number of intersections with the 1-skeleton of the triangulation. 


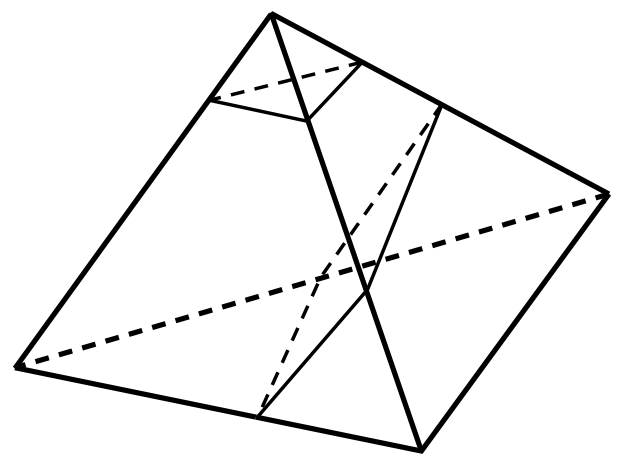

Figure 1.
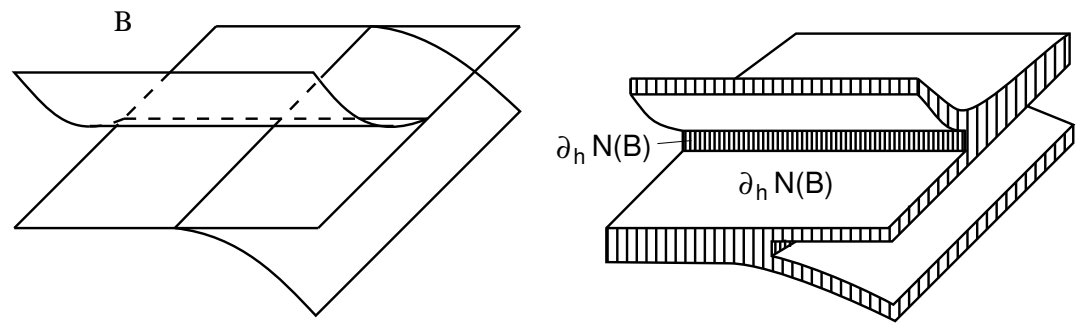

Figure 2.

The transition to branched surfaces is made as follows. Given an essential normal surface of least area in its isotopy class, we push together adjacent discs of the same disc type and identify them. At the boundary of the region where several discs are identified, branching may occur, and we may suppose that the identification space, a branched surface $B \hookrightarrow M$, has a smooth structure which pulls back to a smooth structure on the surface $S$. The locus of points where $B$ is not a manifold is called the branch locus of $B$. The completion of a component of the complement in $B$ of the branch locus is called a sector of $B$. The branched surface just described is called a normal branched surface, since it intersects 3 -simplices in normal discs. It has non-generic branch locus. One can perturb the branch locus by increasing slightly, in a generic manner, the area of identification, and the result is a branched surface with generic branch locus. A branched surface with generic branch locus is locally modelled as shown in Figure 2. It has a 
fibered neighborhood $N(B)$ modelled as shown in Figure 2, which is foliated by interval fibers. The frontier of $N(B)$ is partitioned into the horizontal boundary $\partial_{h} N(B)$ and the vertical boundary $\partial_{v} N(B)$ as shown. There is a similar fibered neighborhood for a branched surface with non-generic branch locus. Clearly, there is a projection map $\pi: N(B) \rightarrow B$ which collapses each fiber. A surface is carried by $B$ if it can be embedded in $N(B)$ transverse to fibers, and it is fully carried if it also intersects every fiber of $N(B)$

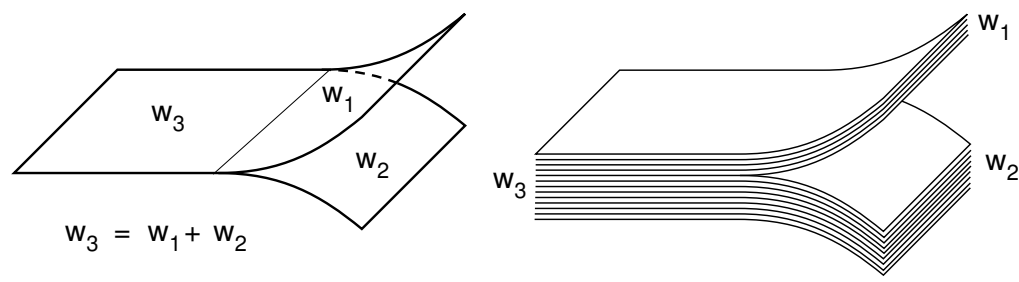

Figure 3.

If $S$ is carried by $B$, it induces a weight vector on $B$. To each sector $Z_{i}$ of $B$ the weight vector assigns the weight $w_{i} \geq 0$ equal to the number of intersections of $\mathrm{S}$ with a fiber $\pi^{-1}(p)$, where $p$ is any point in $\stackrel{\circ}{Z}_{i}$. Furthermore, the weights satisfy branch equations as indicated in Figure 3 . Any weight vector on $B$ satisfying all the branch equations for a branched surface $B$ yields an invariant weight vector on $B$. If the entries of an invariant weight vector $w$ are integers, it is not hard to show that the weight vector determines a surface which we denote $B(w)$. Invariant weight vectors with rationally related weights give weighted surfaces. In general, an invariant weight vector $w$ determines a measured lamination $B(w)$, which we will not describe here in detail. One can think of a measured lamination simply as branched surface with an invariant weight vector. There is an Euler characteristic associated to a measured lamination $B(w)$. Assuming the branched surface is generic, we first assign an Euler characteristic $z_{i}$ to each sector $Z_{i}$. This is not the Euler characteristic of $Z_{i}$ as a compact surface; instead, letting $\left|Z_{i}\right|$ denote the underlying topological surface, $z_{i}=\chi\left(Z_{i}\right)=\chi\left(\left|Z_{i}\right|\right)-(1 / 4) k_{i}$, where $k_{i}$ is the number of "corners" of the sector. The corners can be seen in the local model of Figure 2. Now, we define the Euler characteristic of a measured lamination $B(w)$ as $\chi(B(w))=\sum_{i} z_{i} w_{i}$. This coincides with the usual Euler characteristic of a surface when $w$ is an invariant weight vector with integer entries, see [11].

Given a branched surface $B \hookrightarrow M$ with $s$ sectors, the set of all invariant weight vectors gives a weight cone in $\mathbb{R}^{s}$ which we denote $\mathcal{C}(B)$. It is a cone in the first orthant bounded by finitely many hyperplanes. If we normalize 
invariant weight vectors, requiring for example the sum of entries to equal 1 , then we obtain the weight cell which we denote $\mathcal{M}(B)$. Later in the paper, we will often normalize a weight vector $w$ with integer entries on a normal branched surface by dividing by the area Area $(B(w))$. The normalized weight vectors obtained in this way also lie in a compact set homeomorphic to $\mathcal{M}(B)$. This is because $\sum w_{i}$ differs from $\operatorname{Area}(B(w))$ only by positive scalar multiples, i.e., there exists a constant $C$ such that

$$
(1 / C) \sum w_{i} \leq \operatorname{Area}(B(w)) \leq C \sum w_{i} .
$$

It turns out that the branched surfaces obtained by the construction described above satisfy certain conditions which ensure that any surface carried by the branched surface is essential. We do not need to describe here the exact technical conditions the branched surface satisfies, but we will call a branched surface satisfying these conditions essential. The conditions are those given in [2] to define essential branched surfaces, though essential branched surfaces are there defined for a larger class of 3-manifolds than the class of Haken manifolds. They are also the conditions given in [12] to define Reebless incompressible branched surfaces.

Theorem 2.1 ([12]). Let $M$ be Haken 3-manifold. Then there is a finite collection of essential normal branched surfaces such that every essential surface is fully carried by one of the branched surfaces of the collection as a least area surface.

Theorem $2.2([\mathbf{1 2}])$. Let $M$ be an irreducible, $\partial$-irreducible, 3-manifold and let $B \hookrightarrow M$ be an essential branched surface in $M$. Then any surface carried by $B$ is essential.

\section{Proofs.}

We shall begin with a proof of the following, which easily implies Theorem 1.1.

Theorem 3.1. Let $M$ be a Haken 3-manifold, equipped with a triangulation. Suppose $M$ contains no essential annulus or torus. Then there is a uniform lower bound $K>0$ for the Euler ratio $\mathcal{R}(S)$ on all essential surfaces $S$.

Proof. Suppose there is no uniform lower bound for $\mathcal{R}(S)$, and let $\left\{S_{i}\right\}$ be a sequence of surfaces such that $\mathcal{R}\left(S_{i}\right) \rightarrow 0$. Without loss of generality in view of Theorem 2.1, we may pass to a subsequence and assume that all surfaces $S_{i}$ are fully carried by one essential branched surface $B \hookrightarrow M$. The proof of the Theorem 2.1, see [12], shows that $B$ can be taken to be a normal branched surface, and we can assume that each $S_{i}$ carried by $B$ is a least area normal representative of the isotopy class of $S_{i}$. Each surface $S_{i}$ determines a weight vector $w\left(S_{i}\right)$ on $B$, which we normalize by dividing by $\operatorname{Area}\left(S_{i}\right)$ to obtain $w_{i}=w\left(S_{i}\right) / \operatorname{Area}\left(S_{i}\right)$. These vectors are contained 
in a compact subset of the cone of weight vectors $\mathcal{C}(B)$, hence we may pass to a subsequence and assume $w_{i} \rightarrow w$. The weight vector $w$ determines a measured lamination $B(w)$. Calculating Euler characteristic, which is a linear function on $\mathcal{C}(B)$, we get

$$
\chi(B(w))=\lim _{i \rightarrow \infty} \frac{\chi\left(S_{i}\right)}{\operatorname{Area}\left(S_{i}\right)}=0 .
$$

Thus $w$ is contained in the subset of $\{v \in \mathcal{C}(B): \chi(B(v))=0\}$. The equation $\chi(v)=0$ can be written as a linear equation with integer coefficients in the components $v_{j}$ of $v$, hence there are integer solutions if there are any solutions. We can thus replace $w$ by an integer weight vector $w$ with $\chi(B(w))=0$, and this reperesents a union of annuli and tori. The annuli and tori are essential by Theorem 2.2 , so we have proved the theorem by contradiction.

Next we give a proof of the Theorem 1.4, which implies the theorem of Jaco-Sedgwick. The theorem concerns an irreducible, $\partial$-irreducible 3 manifold $M$ whose boundary is a single torus. As in the introduction, we let $\bar{S}$ denote the surface obtained by capping every boundary component of $S$ by a disc, and we let $\overline{\mathcal{R}}(S)$ denote $|\chi(\bar{S})| / \operatorname{Area}(S)$.

Proof of Theorem 1.4. Suppose there is no uniform lower bound for the Euler ratio $\overline{\mathcal{R}}(S)$ of essential surfaces $S$. Let $\left\{S_{i}\right\}$ be a sequence of surfaces such that $\overline{\mathcal{R}}\left(S_{i}\right) \rightarrow 0$. Without loss of generality in view of Theorem 2.1, we may pass to a subsequence and assume that all surfaces $S_{i}$ are fully carried by one essential branched surface $B \hookrightarrow M$. As before, $S_{i}$ can be taken to be least area normal surfaces carried by the normal branched surface $B$. From Allen Hatcher's result on boundary slopes, [6], we know that all surfaces fully carried by $B$ and with non-empty boundary, have boundary curves of the same slope, $r$ say. Let $\bar{M}$ denote the closed manifold obtained from $M$ using slope $r$ Dehn filling. Then each $S_{i}$ can be capped in $\bar{M}$ by a disc. Furthermore, one can construct a branched surface $\bar{B}$ by attaching a meridian disc of the surgery solid torus to $\partial B$. Note that the attaching map of the disc to $\partial B$ is in general far from an embedding, and there can be highly non-generic branching where the disc is attached. Note also that we do not claim that $\bar{B}$ is essential, though it might be.

Now, as in the previous proof, we use normalized weight vectors $w_{i}=$ $w\left(S_{i}\right) / \operatorname{Area}\left(S_{i}\right)$. Clearly each $w_{i}$ determines a weight vector $\bar{w}_{i}$ on $\bar{B}$ which is the normalized weight vector $\bar{w}_{i}=w\left(\bar{S}_{i}\right) / \operatorname{Area}\left(S_{i}\right)$. As in the previous proof, the weights $\bar{w}_{i}$ are contained in a compact subset of $\mathcal{C}(\bar{B})$, and so after passing to a subsequence, we may assume $\bar{w}_{i} \rightarrow \bar{w}$. As before, we calculate $\chi(\bar{B}(\bar{w}))=0$, and again we can replace $\bar{w}$ by a projectively near weight vector $\bar{w}$ with integer entries. Then $\bar{B}(\bar{w})$ is a union of tori $\bar{T}$ and 
$T=\bar{T} \cap M$ is a union of genus one essential surfaces. The surface $T$ is carried by $B=\bar{B} \cap M$, and is therefore essential by Theorem 2.2 .

Proof of Theorem 1.5. Suppose the theorem is false, and that there is a sequence of surfaces $\left\{S_{n}\right\}$ such that $\overline{\mathcal{R}}\left(S_{n}\right) \rightarrow 0$. Without loss of generality, in view of Theorem 2.1, we may pass to a subsequence and assume that all surfaces $S_{n}$ are fully carried by one essential branched surface $B \hookrightarrow M$. As usual, $S_{n}$ can be taken to be least area normal surfaces carried by the normal branched surface $B$.

In this proof we cannot appeal to Hatcher's theorem on boundary slopes, therefore the analysis is more delicate. Let

$$
\begin{aligned}
& M_{n}=\operatorname{Max}\left\{\operatorname{Length}(b): b \text { is a boundary component of } S_{n}\right\} \\
& m_{n}=\operatorname{Min}\left\{\operatorname{Length}(b): b \text { is a boundary component of } S_{n}\right\} .
\end{aligned}
$$

We begin with easier cases before considering the most general case.

Case 1. There is a uniform bound on $M_{n}$, so that $M_{n} \leq U$ for all $n$. Let $\mathcal{B}\left(S_{n}\right)$ be the set of normal curves in $\partial M$ which occur in $\partial S_{n}$. Then there are just finitely many possibilities for $\mathcal{B}\left(S_{n}\right)$ as $n$ is allowed to vary. Thus we can pass to a subsequence of $\left\{S_{n}\right\}$ and assume that $\mathcal{B}\left(S_{n}\right)=\mathcal{B}$ is the same for all $n$. The set $\mathcal{B}$ can be regarded as an embedded curve system in $\partial M$ carried by $\partial B$ with no two closed curves of $\mathcal{B}$ isotopic in $\partial M$. We can now construct a branched surface $\bar{B}$ with one disc appropriately attached for each curve in $\mathcal{B}$. In fact, $\bar{B}$ is embedded in a 3 -manifold $\bar{M}$ obtained by attaching one 2-handle along $b$ for each curve $b \in \mathcal{B}$. The situation is now very similar to the situation in the proof of Theorem 1.4. Taking $A_{n}=\operatorname{Area}\left(S_{n}\right)$, and passing to a subsequence, we take a limit of the sequence $\left\{\bar{S}_{n} / A_{n}\right\}$ in $\bar{B}$, and the assumption that $\overline{\mathcal{R}}\left(S_{i}\right) \rightarrow 0$ ensures that the limiting weight vector has Euler characteristic 0 and can be approximated by a surface $\bar{T}$ which is a union of tori. Again, the intersection with $M$ must be a union of genus 1 surfaces $T$, and these are essential because they are carried by an essential branched surface. This contradicts the hypotheses of the theorem.

Case 2. The set $\left\{m_{n}: n \geq 1\right\}$ is unbounded. This is, in some sense, the opposite of the previous case. After passing to a subsequence of $\left\{S_{n}\right\}$, we may assume that $m_{n} \rightarrow \infty$. We will again take a limit of a subsequence of $\left\{S_{n} / A_{n}\right\}$, but this time in the branched surface $B$. The limit will again be a measured lamination carried by $B$ (or a limiting weight vector on $B$ ). Each surface $S_{n} / A_{n}$ induces a weight vector on $B$, and the weight vectors are contained in a compact subset of $\mathcal{C}(B)$. In this case, we compare $\chi\left(S_{n} / A_{n}\right)$ to $\chi\left(\bar{S}_{n} / A_{n}\right)$, where $\bar{S}_{n}$ is again the surface with all boundary components capped by discs. (Here the $\bar{S}_{n}$ 's cannot be embedded in any manifold $\bar{M}$.) Since we have normalized, the length of the weighted boundaries $\partial S_{n}$ are 
uniformly bounded, say Length $\left(\partial S_{n} / A_{n}\right)=\left(1 / A_{n}\right) \operatorname{Length}\left(\partial S_{n}\right) \leq R$ for some constant $R$. Now we conclude that

$$
\left|\chi\left(\bar{S}_{n} / A_{n}\right)-\chi\left(S_{n} / A_{n}\right)\right| \leq R / m_{n} \rightarrow 0 .
$$

To see this we note that $\bar{S}_{n} / A_{n}$ is obtained from $S_{n} / A_{n}$ by attaching at most a weight $\operatorname{Length}\left(S_{n} / A_{n}\right) / m_{n}$ of discs. This attachment can change the Euler characteristic by at most $R / m_{n}$ which approaches 0 . Thus $\chi\left(S_{n} / A_{n}\right) \rightarrow$ 0 , since clearly $\chi\left(\bar{S}_{n} / A_{n}\right) \rightarrow 0$. It follows that when we find a limiting lamination by passing to a subsequence and taking limits of weights induced by $S_{n} / A_{n}$ on $B$, we obtain a measured lamination of Euler characteristic 0 in $M$, which can be approximated by a union of tori and annuli. This contradicts the hypotheses of the theorem.

Case 3 . General case. We have an arbitrary sequence $\left\{S_{n}\right\}$ with $\overline{\mathcal{R}}\left(S_{i}\right) \rightarrow 0$. If some normal curve $b_{1}$ occurs as a component of $\partial S_{n}$ for infinitely many $S_{n}$, we pass to a subsequence such that every $S_{n}$ has a boundary component $b_{1}$. In the new sequence $\left\{S_{n}\right\}$, if some normal curve $b_{2} \neq b_{1}$ is a component of $\partial S_{n}$ for infinitely many $n$, we again pass to a subsequence such that $b_{1}$ and $b_{2}$ occur in $\partial S_{n}$ for every $n$. Repeating this argument, we must end with $b_{1}, \ldots, b_{k}$ occurring in $\partial S_{n}$ for all $n$, and with no other normal curve occurring infinitely often.

Let $\hat{M}$ be the manifold obtained by attaching $k$ 2-handles along the curves $b_{1}, \ldots, b_{k}$, and let $\hat{S}_{n}$ denote the surface obtained from $S_{n}$ by capping all curves isotopic to $b_{i}$ by discs in $\hat{M}$. We can assume that all the surfaces $\hat{S}_{n}$ are carried by one branched surface $\hat{B}$, obtained from $B$ by attaching discs using the carrying maps of $b_{i}$ as attaching maps, as in Case 2. Now define $m_{n}=\operatorname{Min}\left\{\operatorname{Length}(b): b\right.$ is a boundary component of $\left.S_{n}, b \neq b_{1}, \ldots, b_{k}\right\}$, or

$$
m_{n}=\operatorname{Min}\left\{\operatorname{Length}(b): b \text { is a boundary component of } \hat{S}_{n}\right\} \text {. }
$$

Clearly $m_{n} \rightarrow \infty$.

As in Case 2, we construct a limiting measured lamination $(\hat{L}, \hat{\mu}) \hookrightarrow \hat{M}$ from $\hat{S}_{n}$ and we show as in Case 2 that $\chi((\hat{L}, \hat{\mu}))=0$. The measured lamination can be approximated by a surface $\hat{S}$ which is a union of annuli and tori in $\hat{M}$. Then we obtain a surface $S=\hat{S} \cap M$ carried by $B$ whose components have genus 1 or genus 0 . The surface $S$ is essential because $B$ is essential. Again, this contradicts the hypotheses of the theorem.

\section{References}

[1] W. Floyd and U. Oertel, Incompressible surfaces via branched surfaces, Topology, 23 (1984), 117-125, MR 85a:57007, Zbl 0524.57008.

[2] D. Gabai and U. Oertel, Essential laminations in 3-manifolds, Ann. of Math., 130 (1989), 41-73, MR 90h:57012, Zbl 0685.57007. 
[3] M. Gromov, Hyperbolic groups, in 'Essays in Group Theory' (S. Gersten, ed.), 8, MSRI Publ., Springer-Verlag, (1987), 75-263, MR 89e:20070, Zbl 0634.20015.

[4] W. Haken, Theorie der normalflächen, Acta. Math., 105 (1961), 245-375, MR 25 \#4519a, Zbl 0100.19402.

[5] A. Hatcher, 3-Manifolds, available on author's web page.

[6] _ On the boundary curves of incompressible surfaces, Pacific J. Math., 99 (1982), 373-377, MR 83h:57016, Zbl 0502.57005.

[7] W. Jaco and U. Oertel, An algorithm to decide if a 3-manifold is a Haken manifold, Topology, 23(2) (1984), 195-209, MR 85j:57014, Zbl 0545.57003.

[8] W. Jaco and J.H. Rubinstein, PL minimal surfaces in 3-manifolds, J. Diff. Geom., 27 (1988), 493-524, MR 89e:57009, Zbl 0652.57005.

[9] H. Kneser, Geschlossene Flächen in dreidimensionalen Mannigfaltigkeiten, Jahres. der Deut. Math. Verein., 38 (1929), 248-260.

[10] J. Morgan and P. Shalen, Degenerations of hyperbolic structures II: Measured laminations in 3-manifolds, Ann. of Math., 127(2) (1988), 403-456, MR 89e:57010a, Zbl 0656.57003.

[11] L. Mosher and U. Oertel, Spaces which are not negatively curved, Comm. in Anal. and Geom., 6 (1991), 67-140, MR 99b:57006, Zbl 0915.53020.

[12] U. Oertel, Measured laminations in 3-manifolds, Trans. Am. Math. Soc., 305(2) (1988), 531-573, MR 89d:57011, Zbl 0652.57006.

[13] J. Plante, A generalization of the Poincaré-Bendixson theorem for foliations of codimension one, Topology, 12 (1973), 177-182, MR 49 \#6253, Zbl 0254.57014.

[14] H. Schubert, Bestimmung der Primfaktorzerlegung von Verkettungen, Math. Z., 76 (1961), 116-148, MR 25 \#4519b, Zbl 0097.16302.

Received May 16, 2000 and revised September 6, 2000. This research was supported by the National Science Foundation.

Department of Mathematics \& Computer Science

Rutgers UNIVERSITY

NEWARK, NJ 07102-3105

E-mail address: oertel@andromeda.rutgers.edu 